\title{
A rapid method for detection of Y-chromosomal DNA from dried blood specimens by the polymerase chain reaction
}

\author{
Michal Witt* and Robert P. Erickson** \\ Department of Human Genetics and Department of Pediatrics and Communicable Diseases, University of Michigan Medical School, \\ Ann Arbor, MI 48109, USA
}

Hum Genet (1989) 82:271-274

The sequence of the Y1 primer that we published has a 1-bp deletion compared to the sequence we actually used. The correct sequence is ATGATAGAAACGGAAATATG. The published sequence has worked successfully in many people's hands.

* Permanent address: Institute of Human Genetics, Polish Academy of Sciences, Poznan, Poland

** Present address: Section of Genetics/Dysmorphology, Department of Pediatrics, College of Medicine, University of Arizona Health Sciences Center, 1501 North Campbell Avenue, Tucson, AZ 85724, USA 